Теорія Ймовір. та Матем. Статист. Вип. 77, 2007
Theor. Probability and Math. Statist.

No. 77, 2008, Pages 15-30 S 0094-9000(09)00744-3

Article electronically published on January 14, 2009

\title{
ON SOME PROPERTIES OF ASYMPTOTIC QUASI-INVERSE FUNCTIONS
}

UDC 519.21

\author{
V. V. BULDYGIN, O. I. KLESOV, AND J. G. STEINEBACH
}

\begin{abstract}
A characterization of normalizing functions connected with the limiting behavior of ratios of asymptotic quasi-inverse functions is discussed. For nondecreasing functions, conditions are obtained that are necessary and sufficient for their asymptotic quasi-inverse functions to belong to the class of (so-called) $O$-regularly varying functions or to some of its subclasses.
\end{abstract}

\section{INTRODUCTION}

This paper is a continuation of Buldygin et al. 10, 11.

Various problems of mathematical analysis and its applications in the theory of probability are connected with the question of finding conditions under which the following implication holds true:

$$
\lim _{t \rightarrow \infty} \frac{g(t)}{f(t)}=a \Longrightarrow \lim _{t \rightarrow \infty} \frac{g^{(-1)}(t)}{f^{(-1)}(t)}=b
$$

where $f(t) \rightarrow \infty$ and $g(t) \rightarrow \infty$ as $t \rightarrow \infty, f^{(-1)}$ and $g^{(-1)}$ are certain "inverse" functions, and $a, b \in[0, \infty]$. This problem was studied, for instance, by Djurčić and Torgašev [16] in the case where $f^{(-1)}$ and $g^{(-1)}$ are inverse or generalized inverse functions, or by Buldygin et al. [8, 11] in the case where $f^{(-1)}$ and $g^{(-1)}$ are inverse or quasi-inverse or asymptotic quasi-inverse functions. A motivation for an application of related characterizations in probability theory is, for example, the correspondence between the strong law of large numbers for random walks and the renewal theorem for counting processes (see, e.g., Gut et al. [20]). Applications to the asymptotic behavior of renewal processes and of solutions of stochastic differential equations were considered in Klesov et al. 25] and Buldygin et al. 8], 11]-13.

A general result (see Buldygin et al. [11]) shows that, under certain conditions,

$$
\lim _{t \rightarrow \infty} \frac{g(t)}{f(t)}=a \in(0, \infty) \Longrightarrow \lim _{t \rightarrow \infty} \frac{g^{\sim}(t)}{f^{\sim}(t / a)}=1,
$$

2000 Mathematics Subject Classification. Primary 26A12; Secondary 26A48.

This work was partially supported by Deutsche Forschungsgemeinschaft under DFG grants 436 UKR 113/41/0-3 and 436 UKR 113/68/0-1. 
where $f^{\sim}$ and $g^{\sim}$ are asymptotic quasi-inverse functions (see the definition below in Section (2). The right-hand side of (2) differs from the right-hand side of (11). If $g$ is a regularly varying function with index $\rho>0$, then the right-hand side of (2) has the following form:

$$
\lim _{t \rightarrow \infty} \frac{g^{\sim}(t)}{f^{\sim}(t)}=\left(\frac{1}{a}\right)^{1 / \rho},
$$

which is similar to the right-hand side in (1).

We prove below (see Remark 3.1) that, under some natural conditions, the relation (11), with $a \neq 1, b \neq 1$, and $f^{(-1)}$ and $g^{(-1)}$ asymptotic quasi-inverse functions, is only possible for normalizing functions $f$ from the so-called class of functions with nondegenerate groups of regular points (see Buldygin et al. [9]).

In this paper we are also concerned with the following question. Let $f$ be a nondecreasing function and let its asymptotic quasi-inverse function $f^{\sim}$ belong to some of the classes of functions studied in the Karamata theory of regular variation or some of its extensions. What are the conditions to be imposed on $f$ to assure certain characteristic properties? The simplest case is when $f$ is continuous, strictly increasing, and belongs to the class of regularly varying functions with positive index. Then it is well known that its inverse function $f^{-1}$ exists and belongs to the same class. In other words, this class of functions is invariant with respect to the transformations $f \mapsto f^{-1}$ and $f^{-1} \mapsto f$. It turns out that there are natural extensions of this class that are invariant with respect to the transformations $f \mapsto f^{\sim}$ and $f^{\sim} \mapsto f$. The descriptions of these classes are obtained here as a byproduct of our main results.

The paper is organized as follows. In Section 2, we recall the definitions and some properties of various classes of regularly varying functions and their extensions. Also in this section, asymptotic quasi-inverse functions are discussed. In Section 3, we study relations between the limits of the ratio of two functions and the limits of the ratio of their quasi-inverse functions. Some known results on the connections of the asymptotic properties of $f$ and $f^{\sim}$, respectively, are discussed in Section 4 . Then, our new results about these connections of $f$ and $f^{\sim}$ are presented; the proofs are given in Section 5 , In Section 6, we briefly introduce asymptotic right quasi-inverses and asymptotic left quasi-inverses and discuss their properties.

\section{Classes of Regularly VARYing FunCtions AND THEIR EXTENSions}

Let $\mathbf{R}$ be the set of real numbers, and $\mathbf{R}_{+}$the set of positive reals. Also let $\mathbb{F}=\mathbb{F}\left(\mathbf{R}_{+}\right)$ be the space of real-valued functions $f=(f(t), t \geq 0)$, and

$$
\mathbb{F}_{+}=\bigcup_{A>0}\{f \in \mathbb{F}: f(t)>0, t \in[A, \infty)\} .
$$

Thus $f \in \mathbb{F}_{+}$if and only if $f$ is eventually positive.

Let $\mathbb{F}^{(\infty)}$ be the space of functions $f \in \mathbb{F}_{+}$such that

(i) $\sup _{0 \leq t \leq T} f(t)<\infty$ for all $T>0$;

(ii) $\lim \sup _{t \rightarrow \infty} f(t)=\infty$.

Furthermore, let $\mathbb{F}^{\infty}$, respectively $\mathbb{F}_{\text {ndec }}^{\infty}$, be the spaces of functions $f \in \mathbb{F}^{(\infty)}$ such that $f(t) \rightarrow \infty$ as $t \rightarrow \infty$, respectively $f$ is nondecreasing for large $t$. We also use the subspace $\mathbb{C}^{\infty}$ of continuous functions in $\mathbb{F}^{\infty}$.

Throughout the paper, "measurability" means "Lebesgue measurability". 
For a given $f \in \mathbb{F}_{+}$, we introduce the upper and lower limit functions

$$
f^{*}(c)=\limsup _{t \rightarrow \infty} \frac{f(c t)}{f(t)} \quad \text { and } \quad f_{*}(c)=\liminf _{t \rightarrow \infty} \frac{f(c t)}{f(t)}, \quad c>0,
$$

which take values in $[0, \infty]$.

2.1. RV functions. In a stimulating paper of 1930, Karamata [22] introduced the notion of regular variation and proved some fundamental theorems for regularly varying functions (see also Karamata [23]). These results (together with later extensions and generalizations) turned out to be fruitful in various fields of mathematics (cf. Seneta 31] and Bingham et al. [7] for excellent surveys on this topic and for the history of the theory and its applications).

Recall that a measurable function $f \in \mathbb{F}_{+}$is called regularly varying (RV) if

$$
f_{*}(c)=f^{*}(c)=\varkappa(c) \in(0, \infty)
$$

for all $c>0$. For any RV function $f, \varkappa(c)=c^{\rho}, c>0$, for some real number $\rho$, which is called the index of the function $f$. The case $\rho=0$ corresponds to a (so-called) slowly varying (SV) function.

Denote by $\mathcal{R V}$ the class of all RV functions, by $\mathcal{R} \mathcal{V}_{\rho}$ the class of all RV functions with index $\rho$, and by $\mathcal{R} \mathcal{V}_{+}$the class of all RV functions with positive index. In particular, $\mathcal{R} \mathcal{V}_{0}$ denotes the class of SV functions.

The boundary case $\rho=\infty$ corresponds to functions $f$ for which

$$
\lim _{t \rightarrow \infty} \frac{f(c t)}{f(t)}=\infty \quad \text { for all } c>1 \text {. }
$$

The functions $f \in \mathcal{R} \mathcal{V}_{\infty}$ are called rapidly varying functions.

2.2. ORV functions. After the work of Karamata, various generalizations of the notion of regular variation appeared in the literature. One of them is a generalization due to Avakumović [2], which was further investigated by Karamata 24], Feller [18, and Aljančić and Arandelović [1. The functions studied by these and several other authors are known in the literature as O-regularly varying (ORV) functions. Bari and Stechkin [3], for example, independently studied ORV functions and their applications in the theory of best function approximation.

A measurable function $f \in \mathbb{F}_{+}$is called $O$-regularly varying if

$$
f^{*}(c)<\infty \text { for all } c>0 .
$$

Recall that monotone ORV functions are known in the literature as dominatedly varying, and a monotone function $f \in \mathbb{F}_{+}$is an ORV function if and only if (4) holds for some $c>1$ (see Feller [18).

2.3. OSV functions. Drasin and Seneta [17] introduced the so-called O-slowly varying (OSV) functions. An ORV function $f$ is called OSV if

$$
\sup _{c>0} f^{*}(c)<\infty .
$$

Let $\mathcal{O S V}$ denote the class of OSV functions. Any slowly varying function $f$ belongs to $\mathcal{O S V}$, since $f^{*}(c)=1, c>0$. OSV functions play an important role in integral representation theorems for functions with a nondegenerate group of regular points (see Buldygin et al. 9]). 
2.4. PRV functions. For any RV function $f$, we have $f^{*}(c) \rightarrow 1$ as $c \rightarrow 1$. This condition is characteristic for a wider class of measurable functions $f \in \mathbb{F}_{+}$, the so-called pseudo-regularly varying functions (PRV). Note that every PRV function is an ORV function, but a quickly varying function is not PRV.

PRV functions and their various applications have been studied by Korenblyum [26], Matuszewska [27, Matuszewska and Orlicz 28, Stadtmüller and Trautner [32, 33], Berman [4, 5], Yakymiv [35, Cline [14], Djurčić [15], Djurčić and Torgašev [16], Klesov et al. 25], and Buldygin et al. 8], 10]-13. Note that the PRV functions are called regularly oscillating in Berman [4, weakly oscillating in Yakymiv [35, intermediate regularly varying in Cline [14, and CRV in Djurčić [15]. We use the term PRV introduced in Buldygin et al. 8 .

One of the well-known properties of PRV functions is that they and only they preserve the equivalence of sequences (cf., e.g., Buldygin et al. [8]). Recall that a function $f$ preserves the equivalence of sequences if

$$
\lim _{n \rightarrow \infty} \frac{f\left(u_{n}\right)}{f\left(v_{n}\right)}=1
$$

for all sequences of positive numbers $\left\{u_{n}\right\}$ and $\left\{v_{n}\right\}$ such that

$$
\lim _{n \rightarrow \infty} u_{n}=\lim _{n \rightarrow \infty} v_{n}=\infty
$$

and $\lim _{n \rightarrow \infty} u_{n} / v_{n}=1$.

Lemma 2.1 ([8]). A measurable function $f \in \mathbb{F}_{+}$preserves the equivalence of sequences if and only if it is PRV.

In what follows we will denote by $\mathcal{O R V}(\mathcal{O S V}, \mathcal{P R} \mathcal{V})$ the set of all ORV (OSV, PRV) functions. Note that

$$
\mathcal{R} \mathcal{V}_{0} \subset \mathcal{R V} \subset \mathcal{P R} \mathcal{O} \subset \mathcal{O} \mathcal{N}
$$

2.5. SQI functions. Let $\mathcal{S} Q \mathcal{J}$ denote the subclass of measurable functions $f \in \mathbb{F}_{+}$satisfying the following condition:

$$
f_{*}(c)>1 \text { for all } c>1 .
$$

We call it the class of sufficiently quickly increasing (SQI) functions. These functions have also been used by Yakymiv [34, Djurčić and Torgašev [16], and Buldygin et al. [8]-[13]. Note that a slowly varying function is not SQI.

2.6. PI functions. Consider the following condition:

$$
f_{*}\left(c_{0}\right)>1 \text { for some } c_{0}>1 \text {. }
$$

Note that condition (6) has been used by Bingham and Goldie [6], de Haan and Stadtmüller [21, Buldygin et al. [8, 11, and Rogozin [30. The functions with property (6) are called positively increasing (PI).

Let $\mathcal{P J}$ be the subclass of measurable functions in $\mathbb{F}_{+}$that satisfy condition (6). Then the following inclusions hold:

$$
\mathcal{R} \mathcal{V}_{\infty} \subset \mathcal{R} \mathcal{V}_{+} \cup \mathcal{R} \mathcal{V}_{\infty} \subset \mathcal{S} Q \mathcal{J} \subset \mathcal{P J}
$$


2.7. Functions with nondegenerate groups of regular points. Consider $f \in \mathbb{F}_{+}$. A number $\lambda>0$ is called a regular point of the function $f$, denoted $\lambda \in \mathbb{G}_{r}(f)$, if $f_{*}(\lambda)=f^{*}(\lambda) \in(0, \infty)$; that is, the limit

$$
\kappa_{f}(\lambda)=\lim _{t \rightarrow \infty} \frac{f(\lambda t)}{f(t)}
$$

exists, and is positive and finite. The set $\mathbb{G}_{r}(f)$ of regular points of $f$ is a multiplicative subgroup of $\mathbf{R}_{+}$with $1 \in \mathbb{G}_{r}(f)$. If $\mathbb{G}_{r}(f)=\{1\}$, then $\mathbb{G}_{r}(f)$ is called degenerate; otherwise, it is nondegenerate.

An ORV function $f$ is called a function with nondegenerate group of regular points if $\mathbb{G}_{r}(f)$ is nondegenerate (cf. Buldygin et al. 9]). It turns out that functions with nondegenerate groups of regular points serve as normalizing sequences in problems connected with partial attraction to certain limit laws (cf., e.g., Grinevich and Khokhlov [19]).

2.8. Asymptotic quasi-inverse functions. The notion of an asymptotic quasi-inverse function is a natural generalization of the notion of an inverse function. Let $f \in \mathbb{F}^{(\infty)}$. If $f$ is continuous and strictly increasing, then its inverse function $f^{-1}$ exists and is determined by the following two properties:

(i) $f\left(f^{-1}(t)\right)=t, t \in\left[f\left(t_{0}\right), \infty\right)$, and

(ii) $f^{-1}(f(t))=t, t \in\left[t_{0}, \infty\right)$.

However, if $f$ is either discontinuous or is not strictly increasing, then its inverse function $f^{-1}$ does not exist. Therefore we consider asymptotic quasi-inverse functions $f^{\sim}$ defined, for a given function $f$, by the properties $f\left(f^{\sim}(t)\right) \sim t$ and $f^{\sim}(t) \rightarrow \infty$ as $t \rightarrow \infty$ (cf. Buldygin et al. [10]). (Here $f(t) \sim g(t)$ as $t \rightarrow \infty$ means that $f(t) / g(t) \rightarrow 1$ as $t \rightarrow \infty$.) That is, we only keep condition (i) in the definition of inverse functions in an asymptotic sense. If, in addition, $f^{\sim}(f(t)) \sim t$ as $t \rightarrow \infty$, then $f^{\sim}$ is called an asymptotic inverse function of $f$ (cf. Bingham et al. [7]). In Section 6, we also introduce asymptotic right quasi-inverse functions, satisfying condition (i) above, and asymptotic left quasi-inverse functions, for which only condition (ii) holds true.

Note that an asymptotic quasi-inverse function is not unique, even when the original function is continuous and strictly increasing. On the other hand, not all functions $f$ have an asymptotic quasi-inverse function. However, for continuous functions $f$, and in various other cases, both their generalized inverse functions $f^{\leftarrow}(s)=\inf \left\{t \in\left[t_{0}, \infty\right): f(t)>s\right\}$ and $\psi(s)=\sup \{t \geq 0: f(t) \leq s\}$, and some other versions of those, are asymptotic quasi-inverse functions (see Buldygin et al. [10]).

The book by Resnick 29] is a standard reference for the properties of generalized inverse functions, in particular in relation to regular variation.

For a given $f$, let $\mathbb{A} \mathbb{Q I}(f)$ be the set of all its asymptotic quasi-inverse functions.

\section{ON LIMITS OF THE RATIO OF FUNCTIONS AND THEIR QUASI-INVERSE FUNCTIONS}

Let $\mathbb{L P}(\varphi)$ be the set of limit points of a function $\varphi$, and let $\mathbb{L} \mathbb{P}\left(\left\{z_{n}\right\}\right)$ be the set of limit points of a sequence $\left\{z_{n}\right\}$. Put

$$
l_{*}\left(c ; f,\left\{z_{n}\right\}\right)=\liminf _{n \rightarrow \infty} \frac{z_{n}}{f\left(c f^{\sim}\left(z_{n}\right)\right)}, \quad l^{*}\left(c ; f,\left\{z_{n}\right\}\right)=\limsup _{n \rightarrow \infty} \frac{z_{n}}{f\left(c f^{\sim}\left(z_{n}\right)\right)}, \quad c>0 .
$$

Lemma 3.1. Let $f, g \in \mathbb{F}^{(\infty)}, f^{\sim} \in \mathbb{A} \mathbb{Q I}(f)$, and $g^{\sim} \in \mathbb{A} \mathbb{Q I}(g)$. Assume that $f$ is $P R V$. If

$$
b \in \mathbb{L} \mathbb{P}\left(g^{\sim} / f^{\sim}\right) \cap \mathbf{R}_{+},
$$

then, for any sequence of positive numbers $\left\{s_{n}\right\}$ such that $s_{n} \rightarrow \infty$ and

$$
\lim _{n \rightarrow \infty} \frac{g^{\sim}\left(s_{n}\right)}{f^{\sim}\left(s_{n}\right)}=b,
$$


one has

$$
l_{*}\left(b ; f,\left\{s_{n}\right\}\right) \in \mathbb{L} \mathbb{P}(g / f), \quad l^{*}\left(b ; f,\left\{s_{n}\right\}\right) \in \mathbb{L} \mathbb{P}(g / f) .
$$

Proof of Lemma 3.1. Since, by Lemma 2.1. the function $f$ preserves the equivalence of sequences, we have, in view of (8) , the following implications:

$$
\begin{aligned}
\lim _{n \rightarrow \infty} \frac{g^{\sim}\left(s_{n}\right)}{b f^{\sim}\left(s_{n}\right)}=1 & \Longrightarrow \lim _{n \rightarrow \infty} \frac{f\left(g^{\sim}\left(s_{n}\right)\right)}{f\left(b f^{\sim}\left(s_{n}\right)\right)}=1 \Longrightarrow \lim _{n \rightarrow \infty} \frac{f\left(b f^{\sim}\left(s_{n}\right)\right)}{f\left(g^{\sim}\left(s_{n}\right)\right)}=1 \\
& \Longrightarrow \lim _{n \rightarrow \infty}\left(\frac{s_{n}}{f\left(g^{\sim}\left(s_{n}\right)\right)}\right)\left(\frac{f\left(b f^{\sim}\left(s_{n}\right)\right)}{s_{n}}\right)=1 \\
& \Longrightarrow \lim _{n \rightarrow \infty}\left(\frac{g\left(t_{n}\right)}{f\left(t_{n}\right)}\right)\left(\frac{f\left(b f^{\sim}\left(s_{n}\right)\right)}{s_{n}}\right)=1,
\end{aligned}
$$

where $t_{n}=g^{\sim}\left(s_{n}\right) \rightarrow \infty$. Thus

$$
\liminf _{n \rightarrow \infty} \frac{g\left(t_{n}\right)}{f\left(t_{n}\right)}=1 / \limsup _{n \rightarrow \infty} \frac{f\left(b f^{\sim}\left(s_{n}\right)\right)}{s_{n}}=l_{*}\left(b ; f,\left\{s_{n}\right\}\right)
$$

and

$$
\limsup _{n \rightarrow \infty} \frac{g\left(t_{n}\right)}{f\left(t_{n}\right)}=1 / \liminf _{n \rightarrow \infty} \frac{f\left(b f^{\sim}\left(s_{n}\right)\right)}{s_{n}}=l^{*}\left(b ; f,\left\{s_{n}\right\}\right) .
$$

This proves Lemma 3.1, since

$$
\liminf _{n \rightarrow \infty} \frac{g\left(t_{n}\right)}{f\left(t_{n}\right)} \in \mathbb{L} \mathbb{P}(g / f), \quad \limsup _{n \rightarrow \infty} \frac{g\left(t_{n}\right)}{f\left(t_{n}\right)} \in \mathbb{L} \mathbb{P}(g / f) .
$$

Corollary 3.1. Let $f, g \in \mathbb{F}^{(\infty)}, f \in \mathcal{P R V}, f^{\sim} \in \mathbb{A} \mathbb{Q} \mathbb{I}(f)$, and let $g^{\sim} \in \mathbb{A} \mathbb{Q} \mathbb{I}(g)$. Then

$$
1 \in \mathbb{L} \mathbb{P}\left(g^{\sim} / f^{\sim}\right) \Longrightarrow 1 \in \mathbb{L} \mathbb{P}(g / f) \text {. }
$$

Corollary 3.2. Let $f, g \in \mathbb{F}^{(\infty)}, f \in \mathcal{P R V}, f^{\sim} \in \mathbb{A} \mathbb{Q I}(f), g^{\sim} \in \mathbb{A} \mathbb{Q I}(g)$, and let $f^{\sim} \in \mathbb{C}^{\infty}$. If the limits

$$
\lim _{t \rightarrow \infty} \frac{g(t)}{f(t)}=a \in \mathbb{R}_{+} \quad \text { and } \quad \lim _{s \rightarrow \infty} \frac{g^{\sim}(s)}{f^{\sim}(s)}=b \in \mathbb{R}_{+}
$$

exist, then also

$$
\lim _{t \rightarrow \infty} \frac{f(t / b)}{f(t)} \in \mathbb{R}_{+}
$$

and

$$
a=\lim _{t \rightarrow \infty} \frac{f(t / b)}{f(t)}
$$

Proof of Corollary 3.2. Note that, by Lemma 3.1 and (9),

$$
l_{*}\left(b ; f,\left\{s_{n}\right\}\right)=l^{*}\left(b ; f,\left\{s_{n}\right\}\right)=\lim _{t \rightarrow \infty} \frac{g(t)}{f(t)}
$$

for any sequence of positive numbers $\left\{s_{n}\right\}$ such that $s_{n} \rightarrow \infty$. This means that the limit

$$
\lim _{t \rightarrow \infty} \frac{t}{f\left(b f^{\sim}(t)\right)}
$$

exists and

But

$$
\lim _{t \rightarrow \infty} \frac{g(t)}{f(t)}=\lim _{t \rightarrow \infty} \frac{t}{f\left(b f^{\sim}(t)\right)}=\lim _{t \rightarrow \infty} \frac{f\left(f^{\sim}(t)\right)}{f\left(b f^{\sim}(t)\right)}
$$

$$
\lim _{t \rightarrow \infty} \frac{f\left(f^{\sim}(t)\right)}{f\left(b f^{\sim}(t)\right)}=\lim _{t \rightarrow \infty} \frac{f(t)}{f(b t)}=\lim _{t \rightarrow \infty} \frac{f(t / b)}{f(t)}
$$


if $f^{\sim} \in \mathbb{C}^{\infty}$. Thus (10) and (11) are proved.

The following theorem is similar to Corollary 3.2, but includes other conditions on the functions $f$ and $f^{\sim}$.

Theorem 3.1. Let $f \in \mathbb{F}_{\text {ndec }}^{\infty}, g \in \mathbb{F}^{(\infty)}, f^{\sim} \in \mathbb{A} \mathbb{Q I}(f)$, and $g^{\sim} \in \mathbb{A} \mathbb{Q I}(g)$. Assume that $f$ is PRV and $f^{\sim}$ is nondecreasing. If (9) holds, then (10) and (11) follow.

For the proof of Theorem 3.1 we need the following result.

Lemma 3.2. Let $f \in \mathbb{F}_{\text {ndec }}^{\infty}$ and $f^{\sim} \in \mathbb{A} \mathbb{Q I}(f)$. Assume that $f^{\sim}$ is nondecreasing. Then, for some $c_{0}>0$, the limit

$$
l_{1}\left(c_{0}\right)=\lim _{t \rightarrow \infty} \frac{f\left(c_{0} f^{\sim}(t)\right)}{t} \in[0, \infty]
$$

exists if and only if the limit

$$
l_{2}\left(c_{0}\right)=\lim _{t \rightarrow \infty} \frac{f\left(c_{0} t\right)}{f(t)} \in[0, \infty]
$$

exists. In the latter case, $l_{1}\left(c_{0}\right)=l_{2}\left(c_{0}\right)$.

Proof of Lemma 3.2. First assume that the limit $l_{2}\left(c_{0}\right)$ exists. Then

$$
\lim _{t \rightarrow \infty} \frac{f\left(c_{0} t\right)}{f(t)}=\lim _{t \rightarrow \infty} \frac{f\left(c_{0} f^{\sim}(t)\right)}{f\left(f^{\sim}(t)\right)}=\lim _{t \rightarrow \infty} \frac{f\left(c_{0} f^{\sim}(t)\right)}{t},
$$

that is, the limit $l_{1}\left(c_{0}\right)$ exists and $l_{1}\left(c_{0}\right)=l_{2}\left(c_{0}\right)$.

Now assume that $l_{1}\left(c_{0}\right)$ exists. Consider a sequence of positive numbers $\left\{s_{n}\right\}$ tending to $\infty$. Since $f^{\sim}$ is nondecreasing, for any $n \geq 1$ there exist positive numbers $t_{n}$ and $\tau_{n}$ such that $t_{n} \rightarrow \infty, t_{n} / \tau_{n} \rightarrow 1$, and $f^{\sim}\left(t_{n}\right) \leq s_{n} \leq f^{\sim}\left(\tau_{n}\right)$. Then

$$
f^{\sim}\left(t_{n}\right) \leq s_{n} \leq f^{\sim}\left(\tau_{n}\right)
$$

Hence

$$
\begin{aligned}
l_{1}\left(c_{0}\right) & =\liminf _{n \rightarrow \infty} \frac{f\left(c_{0} f^{\sim}\left(t_{n}\right)\right)}{t_{n}}=\liminf _{n \rightarrow \infty} \frac{f\left(c_{0} f^{\sim}\left(t_{n}\right)\right)}{\tau_{n}}=\liminf _{n \rightarrow \infty} \frac{f\left(c_{0} f^{\sim}\left(t_{n}\right)\right)}{f\left(f^{\sim}\left(\tau_{n}\right)\right)} \\
& \leq \liminf _{n \rightarrow \infty} \frac{f\left(c_{0} s_{n}\right)}{f\left(s_{n}\right)} \leq \limsup _{n \rightarrow \infty} \frac{f\left(c_{0} s_{n}\right)}{f\left(s_{n}\right)} \leq \limsup _{n \rightarrow \infty} \frac{f\left(c_{0} f^{\sim}\left(\tau_{n}\right)\right)}{f\left(f^{\sim}\left(t_{n}\right)\right)} \\
& =\limsup _{n \rightarrow \infty} \frac{f\left(c_{0} f^{\sim}\left(\tau_{n}\right)\right)}{t_{n}}=\limsup _{n \rightarrow \infty} \frac{f\left(c_{0} f^{\sim}\left(\tau_{n}\right)\right)}{\tau_{n}}=l_{1}\left(c_{0}\right),
\end{aligned}
$$

since $f$ is nondecreasing. This means that, for any sequence of positive numbers $\left\{s_{n}\right\}$ tending to $\infty$,

$$
\lim _{n \rightarrow \infty} \frac{f\left(c_{0} s_{n}\right)}{f\left(s_{n}\right)}=l_{1}\left(c_{0}\right) .
$$

Hence $l_{2}\left(c_{0}\right)$ exists and $l_{1}\left(c_{0}\right)=l_{2}\left(c_{0}\right)$.

This completes the proof of Lemma 3.2

Proof of Theorem 3.1. It is the same as the proof of Corollary [3.2, just making use of Lemma 3.2 in (12).

Remark 3.1. Assume the conditions of Corollary 3.2 or Theorem 3.1 Then (10) and (11) hold true. If (9) holds with $b=1$, then (10) is trivial and, by (9) and (11), one has

$$
\lim _{t \rightarrow \infty} \frac{g(t)}{f(t)}=\lim _{t \rightarrow \infty} \frac{g^{\sim}(t)}{f^{\sim}(t)}=1
$$


But, if $b>0$ and $b \neq 1$, then (10) is nontrivial, and in this case $f$ is a function with a nondegenerate group of regular points (see Section 21). Note also that, by (11), $b \neq 1$ if $a \neq 1$.

Assume that (9) holds with $b \neq 1$. Then $1 / b \in \mathbb{G}_{r}(f)$ (see Section 2) and

$$
\lim _{t \rightarrow \infty} \frac{g(t)}{f(t)}=a=\lim _{t \rightarrow \infty} \frac{f(t / b)}{f(t)}=\left(\frac{1}{b}\right)^{\rho},
$$

where $\rho$ denotes the index of $f$, i.e.,

$$
\rho=\lim _{\lambda \rightarrow \infty} \frac{\log f^{*}(\lambda)}{\log \lambda}=\lim _{t \rightarrow \infty} \frac{\log f(t)}{\log t}
$$

(see Theorem 5.1, Corollary 5.3, and Corollary 7.2 in Buldygin et al. 9]). If $\rho>0$, then

$$
\lim _{t \rightarrow \infty} \frac{g^{\sim}(t)}{f^{\sim}(t)}=b=\left(\frac{1}{a}\right)^{1 / \rho}
$$

(cf. equality (3) ).

\section{Connections Between the asymptotic properties of $f$ And $f^{\sim}$}

In the sequel, we assume that $f \in \mathbb{F}^{(\infty)}, f^{\sim}$ is an asymptotic quasi-inverse function of $f$, and both $f$ and $f^{\sim}$ are measurable functions.

4.1. Some earlier results. It is well known that, for any $\rho>0$,

$$
f \in \mathcal{R} \mathcal{V}_{1 / \rho} \Longleftrightarrow f^{\sim} \in \mathcal{R} \mathcal{V}_{\rho}
$$

and if $f \in \mathcal{R} \mathcal{V}_{+}$, then $f^{\sim}$ is an asymptotic inverse function of $f$ (see, e.g., Bingham et al. [7, Theorem 1.5.12, and Buldygin et al. [11, Theorem 8.4). One can rewrite this assertion in a different form:

$$
f \in \mathcal{R} \mathcal{V}_{+} \Longleftrightarrow f^{\sim} \in \mathcal{R} \mathcal{V}_{+} .
$$

In other words, asymptotic quasi-inverse functions are in the same class as their originals.

If $f$ is nondecreasing, then

$$
f \in \mathcal{S Q J} \Longrightarrow f^{\sim} \in \mathcal{P R} \mathcal{V},
$$

and if, in addition, $f^{\sim}$ is an asymptotic inverse function of $f$, then

$$
f \in \mathcal{S} Q \mathcal{J} \Longleftrightarrow f^{\sim} \in \mathcal{P R} \mathcal{V}
$$

(cf. Buldygin et al. 10, Proposition 7.1 and Theorem 7.1; for continuous functions $f$, see also Buldygin et al. 8], Proposition 6.1). On the other hand, if either $f$ or $f^{\sim}$ is nondecreasing, then

$$
f \in \mathcal{P R} \mathcal{C} \Longrightarrow f^{\sim} \in \mathcal{S} \mathcal{Q} \mathcal{J},
$$

and if $f^{\sim}$ is an asymptotic inverse function of $f$ and $f$ is nondecreasing, then

$$
f \in \mathcal{P R} \mathcal{2} \Longleftrightarrow f^{\sim} \in \mathcal{S} Q \mathcal{J}
$$

(cf. Buldygin et al. [11, Proposition 8.1, and Buldygin et al. 10, Corollary 7.1; for increasing and continuous functions $f$, see also Buldygin et al. [8]).

The following statement is similar to (13):

$$
f \in \mathcal{S} Q \mathcal{J} \cap \mathcal{P R V} \Longleftrightarrow f^{\sim} \in \mathcal{S} Q \mathcal{J} \cap \mathcal{P R} \mathcal{V},
$$

and, if $f \in \mathcal{S} Q \mathcal{J} \cap \mathcal{P R} \mathcal{V}$, then $f^{\sim}$ is an asymptotic inverse function of $f$ (cf. Buldygin et al. [11, Theorem 8.4; for increasing and continuous functions $f$, see also Djurčić and Torgašev [16], and Buldygin et al. 8] ).

The corresponding characterizations are open for the classes $\mathcal{O} \mathcal{R} \mathcal{V}, \mathcal{R} \mathcal{V}_{0}, \mathcal{R} \mathcal{V}_{\infty}$, and some other related classes such as $\mathcal{O S V}$. 
4.2. Some new results. The following theorems hold true.

Theorem 4.1. Let $f$ be nondecreasing. Then

$$
f \in \mathcal{P J} \Longrightarrow f^{\sim} \in \mathcal{O} \mathcal{R} \mathcal{V} .
$$

Theorem 4.2. Let both functions $f$ and $f^{\sim}$ be nondecreasing. Then

(i) $f \in \mathcal{P J} \Longleftrightarrow f^{\sim} \in \mathcal{O} \mathcal{R V}$;

(ii) $f \in \mathcal{O} \mathcal{R V} \Longleftrightarrow f^{\sim} \in \mathcal{P J}$.

Theorem 4.3. Let both functions $f$ and $f^{\sim}$ be nondecreasing. Then

(iii) $f \in \mathcal{R} \mathcal{V}_{\infty} \Longleftrightarrow f^{\sim} \in \mathcal{R} \mathcal{V}_{0}$;

(iv) $f \in \mathcal{R} \mathcal{V}_{0} \Longleftrightarrow f^{\sim} \in \mathcal{R} \mathcal{V}_{\infty}$.

The last two theorems provide characterizations for the classes $\mathcal{R} \mathcal{V}_{0}, \mathcal{R} \mathcal{V}_{\infty}, \mathcal{O} \mathcal{R} \mathcal{V}$, and PJ. The next result follows from Theorem 4.2,

Theorem 4.4. Let both functions $f$ and $f^{\sim}$ be nondecreasing. Then

$$
f \in \mathcal{O} \mathcal{R V} \cap \mathcal{P J} \Longleftrightarrow f^{\sim} \in \mathcal{O} \mathcal{R V} \cap \mathcal{P J} \text {. }
$$

The intersection of the corresponding classes in (5) and (7) gives

$$
\mathcal{R} \mathcal{V}_{+} \subset \mathcal{P R V} \cap \mathcal{S Q J} \subset \mathcal{O R} \mathcal{P} \cap \mathcal{P J}
$$

where all the classes $\mathcal{R} \mathcal{V}_{+}, \mathcal{P R V} \cap \mathcal{S} \mathcal{O J}$, and $\mathcal{O} \mathcal{R V} \cap \mathcal{P J}$ are invariant with respect to both transformations $f \mapsto f^{\sim}$ and $f^{\sim} \mapsto f$.

Next we turn to the class of OSV functions. Consider the following condition:

$$
\lim _{t \rightarrow \infty} \frac{f\left(c_{0} f^{\sim}(t)\right)}{t}=\infty \text { for some } c_{0}>1 .
$$

It is obvious that (14) is equivalent to

$$
\lim _{t \rightarrow \infty} \frac{f\left(c_{0} t\right)}{f(t)}=\infty \quad \text { for some } c_{0}>1
$$

if $f^{\sim}$ is continuous. It is less obvious that (14) and (15) are equivalent if both functions $f$ and $f^{\sim}$ are nondecreasing; see Lemma 3.2 .

Let $Q \mathcal{V}$ and $Q \mathcal{V}_{1}$ be the subclasses of measurable functions of $\mathbb{F}^{(\infty)}$ satisfying conditions (14) and (15), respectively.

Theorem 4.5. Let $f$ be nondecreasing. Then $f \in \mathcal{Q V} \Longleftrightarrow f^{\sim} \in \mathcal{O S V}$.

Theorem 4.6. Let both functions $f$ and $f^{\sim}$ be nondecreasing. Then

(v) $f \in \mathcal{Q} \mathcal{V}_{1} \Longleftrightarrow f^{\sim} \in \mathcal{O S V}$;

(vi) $f \in \mathcal{O S V} \Longleftrightarrow f^{\sim} \in \mathcal{Q} \mathcal{V}_{1}$.

\section{Proof of Theorems 4.1-4.6}

Proof of Theorem 4.1. First assume that $f \in \mathcal{P J}$, but $f^{\sim} \notin \mathcal{O} \mathcal{R V}$. Since $f^{\sim} \notin \mathcal{O} \mathcal{R V}$, there exists a number $a>0$ such that

$$
\left(f^{\sim}\right)^{*}(a)=\limsup _{t \rightarrow \infty} \frac{f^{\sim}(a t)}{f^{\sim}(t)}=\infty .
$$

Hence, for any $\varepsilon>0$, there is an $a=a(\varepsilon) \in(0,1+\varepsilon)$ such that (16) holds, since $\left(f^{\sim}\right)^{*}(\sqrt{u}) \geq \sqrt{\left(f^{\sim}\right)^{*}(u)}$ for every $u>1$. This means that, for any $\varepsilon>0$, there exist a number $a=a(\varepsilon) \in(0,1+\varepsilon)$ and a sequence of positive numbers $\left\{s_{n}\right\}$, increasing to $\infty$, 
such that, for any $A>0$,

$$
f^{\sim}\left(a(\varepsilon) s_{n}\right) \geq A f^{\sim}\left(s_{n}\right)
$$

for sufficiently large $n$.

Moreover, since $f \in \mathcal{P J}$, there exists a number $\delta>0$ such that, for any sequence of positive numbers $\left\{t_{n}\right\}$ tending to $\infty$,

$$
f\left(c_{0} t_{n}\right)>(1+\delta) f\left(t_{n}\right)
$$

for sufficiently large $n$, where $c_{0}$ is the constant from condition (6).

Now, by relations (17) and (18), for $\varepsilon=\delta$ and $A=c_{0}$, we have

$$
\begin{aligned}
1+\delta & >a(\delta)=\lim _{n \rightarrow \infty} \frac{f\left(f^{\sim}\left(a(\delta) s_{n}\right)\right)}{s_{n}} \geq \limsup _{n \rightarrow \infty} \frac{f\left(c_{0} f^{\sim}\left(s_{n}\right)\right)}{s_{n}} \\
& \geq(1+\delta) \lim _{n \rightarrow \infty} \frac{f\left(f^{\sim}\left(s_{n}\right)\right)}{s_{n}}=1+\delta .
\end{aligned}
$$

This contradiction proves Theorem 4.1 .

The proof of Theorem 4.2 makes use of the following auxiliary result.

Lemma 5.1. Let both functions $f$ and $f^{\sim}$ be nondecreasing. Then

$$
f^{\sim} \in \mathcal{O} \mathcal{R V} \Longrightarrow f \in \mathcal{P J} \text {. }
$$

Proof of Lemma 5.1. Let $f^{\sim} \in \mathcal{O} \mathcal{R} \mathcal{V}$, but $f \notin \mathcal{P J}$. Since $f^{\sim} \in \mathcal{O} \mathcal{R} \mathcal{V}$, for any fixed $a>1$, there exists a number $A>1$ such that

$$
f^{\sim}(a s) \leq A f^{\sim}(s)
$$

for sufficiently large $s$. Moreover, there is a sequence of positive numbers $\left\{s_{n}\right\}$, strictly increasing to $\infty$, such that

$$
\lim _{n \rightarrow \infty} \frac{f\left(A s_{n}\right)}{f\left(s_{n}\right)}=1,
$$

since $f \notin \mathcal{P J}$ and $f$ is nondecreasing.

Put $t_{n}=\sup \left\{t \geq 0: f^{\sim}(t) \leq s_{n}\right\}$ and $\tau_{n}=\inf \left\{t \geq 0: f^{\sim}(t)>s_{n}\right\}, n \geq 1$ (here $\sup \varnothing:=0)$. Then $t_{n} \rightarrow \infty$ and $t_{n}=\tau_{n}, n \geq 1$, since $f^{\sim}$ is nondecreasing and unbounded.

Hence, for $u_{n}=t_{n}-1$ and $v_{n}=t_{n}+1, n \geq 1$, we have

$$
f^{\sim}\left(u_{n}\right) \leq s_{n} \leq f^{\sim}\left(v_{n}\right), \quad n \geq 1,
$$

and

$$
u_{n} \sim f\left(s_{n}\right) \sim v_{n}, \quad n \rightarrow \infty,
$$

since $f^{\sim}$ and $f$ are nondecreasing.

Thus, by (19)-(22),

$$
\begin{aligned}
1 & <a=\lim _{n \rightarrow \infty} \frac{f\left(f^{\sim}\left(a u_{n}\right)\right)}{u_{n}} \leq \limsup _{n \rightarrow \infty} \frac{f\left(A f^{\sim}\left(u_{n}\right)\right)}{u_{n}} \leq \limsup _{n \rightarrow \infty} \frac{f\left(A s_{n}\right)}{u_{n}} \\
& =\limsup _{n \rightarrow \infty} \frac{f\left(A s_{n}\right)}{f\left(s_{n}\right)} \frac{f\left(s_{n}\right)}{u_{n}}=\lim _{n \rightarrow \infty} \frac{f\left(A s_{n}\right)}{f\left(s_{n}\right)}=1 .
\end{aligned}
$$

This contradiction proves Lemma 5.1

Proof of Theorem 4.2. Statement (i) follows from a combination of Theorem 4.1 and Lemma 5.1 .

Next we prove statement (ii). First we assume that $f \notin \mathcal{O} \mathcal{R} \mathcal{V}$, but $f^{\sim} \in \mathcal{P J}$. Since $f^{\sim} \in \mathcal{P J}$, there exist numbers $c_{0}>1$ and $b>1$ such that

$$
f^{\sim}\left(c_{0} t\right) \geq b f^{\sim}(t)
$$


for sufficiently large $t$. Since $f$ is nondecreasing, $f^{*}(a)=\infty$ for all $a>1$, as in the proof of Theorem 4.1 Thus, given constants $a>1$ and $A>1$, there is an increasing sequence $\left\{s_{n}\right\}$ such that $s_{n} \rightarrow \infty$ as $n \rightarrow \infty$ and

$$
f\left(a s_{n}\right) \geq A f\left(s_{n}\right), \quad n \geq 1 .
$$

Now, we define sequences $\left\{u_{n}\right\}$ and $\left\{v_{n}\right\}$ as in the proof of Lemma 5.1 and choose $a=b$ and $A=2 c_{0}$. Then

$$
\begin{aligned}
c_{0} & =\lim _{n \rightarrow \infty} \frac{f\left(f^{\sim}\left(c_{0} v_{n}\right)\right)}{v_{n}} \geq \limsup _{n \rightarrow \infty} \frac{f\left(b f^{\sim}\left(v_{n}\right)\right)}{v_{n}} \geq \limsup _{n \rightarrow \infty} \frac{f\left(b s_{n}\right)}{v_{n}} \\
& \geq 2 c_{0} \limsup _{n \rightarrow \infty} \frac{f\left(s_{n}\right)}{v_{n}}=2 c_{0} .
\end{aligned}
$$

This contradiction proves that $f \in \mathcal{O} \mathcal{R V}$.

Assume now that $f \in \mathcal{O} \mathcal{R} \mathcal{V}$, but $f^{\sim} \notin \mathcal{P J}$. Since $f \in \mathcal{O} \mathcal{R} \mathcal{V}$, for any $a>1$, there exists an $A>1$ such that

$$
f(a t) \leq A f(t)
$$

for sufficiently large $t$. At the same time, since $f^{\sim} \notin \mathcal{P J}$, for any $c>1$ and any number $b>1$, there exists a sequence of positive numbers $\left\{s_{n}\right\}$, strictly increasing to $\infty$, such that

$$
f^{\sim}\left(c s_{n}\right) \leq b f^{\sim}\left(s_{n}\right), \quad n \geq 1 .
$$

For a fixed $a>1$, choose $b=a$ and $c=2 A$. Then

$$
2 A \leq \lim _{n \rightarrow \infty} \frac{f\left(f^{\sim}\left(c s_{n}\right)\right)}{s_{n}} \leq \limsup _{n \rightarrow \infty} \frac{f\left(a f^{\sim}\left(s_{n}\right)\right)}{s_{n}} \leq A \limsup _{n \rightarrow \infty} \frac{f\left(f^{\sim}\left(s_{n}\right)\right)}{s_{n}}=A .
$$

This contradiction proves the implication $f^{\sim} \in \mathcal{O} \mathcal{R V} \Longrightarrow f \in \mathcal{P J}$, which completes the proof of (ii).

Proof of Theorem 4.3. We first prove statement (iii). Assume that $f \in \mathcal{R} \mathcal{V}_{\infty}$, but $f^{\sim} \notin \mathcal{R} \mathcal{V}_{0}$. Since $f^{\sim} \notin \mathcal{R} \mathcal{V}_{0}$, there exist numbers $c_{0}>1, a>1$, and a sequence of positive numbers $\left\{t_{n}\right\}$, strictly increasing to $\infty$, such that

$$
f^{\sim}\left(c_{0} t_{n}\right) \geq a f^{\sim}\left(t_{n}\right), \quad n \geq 1 .
$$

Thus, since $f \in \mathcal{R} \mathcal{V}_{\infty}$,

$$
\infty=\lim _{n \rightarrow \infty} \frac{f\left(a f^{\sim}\left(t_{n}\right)\right)}{f\left(f^{\sim}\left(t_{n}\right)\right)} \leq \lim _{n \rightarrow \infty} \frac{f\left(f^{\sim}\left(c_{0} t_{n}\right)\right)}{f\left(f^{\sim}\left(t_{n}\right)\right)}=c_{0} .
$$

This contradiction proves the implication $f \in \mathcal{R} \mathcal{V}_{\infty} \Longrightarrow f^{\sim} \in \mathcal{R} \mathcal{V}_{0}$.

Now assume that $f^{\sim} \in \mathcal{R} \mathcal{V}_{0}$, but $f \notin \mathcal{R} \mathcal{V}_{\infty}$. Since $f^{\sim} \in \mathcal{R} \mathcal{V}_{0}$, for all $c>1$ and all $a>1$,

for sufficiently large $t$.

$$
f^{\sim}(c t) \leq a f^{\sim}(t)
$$

Moreover, as $f \notin \mathcal{R} \mathcal{V}_{\infty}$, there exist numbers $\lambda>1$ and $A>0$, and a sequence $\left\{s_{n}\right\}$, tending to $\infty$, such that

$$
f\left(\lambda s_{n}\right) \leq A f\left(s_{n}\right), \quad n \geq 1 .
$$

Choose $a=\lambda, c>A$, and sequences $\left\{u_{n}\right\}$ and $\left\{v_{n}\right\}$ as in the proof of Lemma 5.1. Then

$$
\begin{aligned}
c & =\lim _{n \rightarrow \infty} \frac{f\left(f^{\sim}\left(c u_{n}\right)\right)}{u_{n}} \leq \limsup _{n \rightarrow \infty} \frac{f\left(a f^{\sim}\left(u_{n}\right)\right)}{u_{n}} \leq \limsup _{n \rightarrow \infty} \frac{f\left(a s_{n}\right)}{u_{n}} \\
& \leq A \limsup _{n \rightarrow \infty} \frac{f\left(s_{n}\right)}{u_{n}}=A .
\end{aligned}
$$

This contradiction completes the proof of (iii). 
Next we prove statement (iv). First assume that $f \in \mathcal{R} \mathcal{V}_{0}$, but $f^{\sim} \notin \mathcal{R} \mathcal{V}_{\infty}$. Since $f^{\sim} \notin \mathcal{R} \mathcal{V}_{\infty}$, there are numbers $c>1, a>0$, and a sequence of positive numbers $\left\{t_{n}\right\}$, strictly increasing to $\infty$, such that

$$
f^{\sim}\left(c t_{n}\right) \leq a f^{\sim}\left(t_{n}\right), \quad n \geq 1
$$

whence $f\left(f^{\sim}\left(c t_{n}\right)\right) \leq f\left(a f^{\sim}\left(t_{n}\right)\right)$ for all $n \geq 1$. Moreover, since $f \in \mathcal{R} \mathcal{V}_{0}$,

$$
1<c=\lim _{n \rightarrow \infty} \frac{f\left(f^{\sim}\left(c t_{n}\right)\right)}{t_{n}} \leq \lim _{n \rightarrow \infty} \frac{f\left(a f^{\sim}\left(t_{n}\right)\right)}{t_{n}}=\lim _{n \rightarrow \infty} \frac{f\left(f^{\sim}\left(t_{n}\right)\right)}{t_{n}}=1 .
$$

This contradiction proves the implication $f \in \mathcal{R} \mathcal{V}_{0} \Longrightarrow f^{\sim} \in \mathcal{R} \mathcal{V}_{\infty}$.

Now assume that $f^{\sim} \in \mathcal{R} \mathcal{V}_{\infty}$, but $f \notin \mathcal{R} \mathcal{V}_{0}$. Since $f \notin \mathcal{R} \mathcal{V}_{0}$, there exist $c_{0}>1, a>1$, and a sequence of positive numbers $\left\{s_{n}\right\}$, strictly increasing to $\infty$, such that

$$
f\left(c_{0} s_{n}\right) \geq a f\left(s_{n}\right), \quad n \geq 1 .
$$

Let $c>c_{0}$. Then, since $f^{\sim} \in \mathcal{R} \mathcal{V}_{\infty}$, for every $\lambda>1$

$$
f^{\sim}(\lambda t) \geq c f^{\sim}(t) .
$$

Choose two sequences of positive numbers $\left\{u_{n}\right\}$ and $\left\{v_{n}\right\}$, increasing to $\infty$, such that relations (21) and (22) hold (confer the proof of Lemma 5.1 for details). On account of (24), for $t=v_{n}$, and using (21) and (23), we get

$$
f\left(f^{\sim}\left(\lambda v_{n}\right)\right) \geq f\left(c f^{\sim}\left(v_{n}\right)\right) \geq f\left(c_{0} s_{n}\right) \geq a f\left(s_{n}\right)
$$

for sufficiently large $n$. Thus, by (22),

$$
1=\limsup _{n \rightarrow \infty} \frac{f\left(s_{n}\right)}{v_{n}}=\limsup _{n \rightarrow \infty} \frac{\lambda f\left(s_{n}\right)}{f\left(f^{\sim}\left(\lambda v_{n}\right)\right)} \leq \frac{\lambda}{a}
$$

for any $\lambda>1$. Letting $\lambda \downarrow 1$ we get a contradiction. This proves the implication $f^{\sim} \in \mathcal{R} \mathcal{V}_{\infty} \Longrightarrow f \in \mathcal{R} \mathcal{V}_{0}$ and completes the proof of (iv).

Proof of Theorem 4.5. Let $f^{\sim} \in \mathcal{O S V}$. Then there exists a number $A>0$ such that $f^{\sim}(c t) \leq A f^{\sim}(t)$ for all $c>0$ and sufficiently large $t$, whence $f\left(f^{\sim}(c t)\right) \leq f\left(A f^{\sim}(t)\right)$ and

$$
c=\lim _{t \rightarrow \infty} \frac{f\left(f^{\sim}(c t)\right)}{t} \leq \liminf _{t \rightarrow \infty} \frac{f\left(A f^{\sim}(t)\right)}{t} .
$$

Since $c$ is arbitrary, (14) holds with $c_{0}=A$. Thus $f \in \mathcal{Q V}$ and the implication

$$
f^{\sim} \in \mathcal{O S V} \Longrightarrow f \in \mathcal{Q} \mathcal{V}
$$

is proved.

Now assume that $f \in \mathcal{Q} \mathcal{V}$, but $f^{\sim} \notin \mathcal{O} \mathcal{S} \mathcal{V}$. Then, for any $A>0$, there exist a number $c>0$ and a sequence of positive numbers $\left\{t_{n}\right\}$ tending to $\infty$, such that

$$
f^{\sim}\left(c t_{n}\right) \geq A f^{\sim}\left(t_{n}\right), \quad n \geq 1 .
$$

In particular, for $A=c_{0}$, there exist $c>0$ and $\left\{t_{n}\right\}$ such that $f^{\sim}\left(c t_{n}\right) \geq c_{0} f^{\sim}\left(t_{n}\right), n \geq 1$, whence $f\left(f^{\sim}\left(c t_{n}\right)\right) \geq f\left(c_{0} f^{\sim}\left(t_{n}\right)\right), n \geq 1$, and

$$
c=\lim _{n \rightarrow \infty} \frac{f\left(f^{\sim}\left(c t_{n}\right)\right)}{t_{n}} \geq \liminf _{n \rightarrow \infty} \frac{f\left(c_{0} f^{\sim}\left(t_{n}\right)\right)}{t_{n}} \geq \liminf _{t \rightarrow \infty} \frac{f\left(c_{0} f^{\sim}(t)\right)}{t},
$$

which contradicts the assumption that $f \in \mathcal{Q V}$. Thus $f^{\sim} \in \mathcal{O S} \mathcal{V}$ and the implication $f \in \mathcal{Q V} \Longrightarrow f^{\sim} \in \mathcal{O S V}$ is proved. 
Proof of Theorem 4.6. Statement (v) follows from a combination of Theorem 4.5 and Lemma 3.2 .

Let $f^{\sim} \in \mathcal{Q} \mathcal{V}_{1}$. Then there exists a number $c_{0}$ such that

$$
\lim _{t \rightarrow \infty} \frac{f^{\sim}\left(c_{0} t\right)}{f^{\sim}(t)}=\infty .
$$

Thus, for every $A>0$,

$$
f^{\sim}\left(c_{0} t\right) \geq A f^{\sim}(t)
$$

for all sufficiently large $t$.

Since $f$ is nondecreasing,

$$
c_{0}=\lim _{t \rightarrow \infty} \frac{f\left(f^{\sim}\left(c_{0} t\right)\right)}{t} \geq \limsup _{t \rightarrow \infty} \frac{f\left(A f^{\sim}(t)\right)}{t}
$$

for all $A>0$. Next we show that

$$
\sup _{A>0} f^{*}(A) \leq c_{0}
$$

Otherwise, there is an $A_{0}>0$ such that $f^{*}\left(A_{0}\right)>c_{0}$. Pick $B$ with $c_{0}<B<f^{*}\left(A_{0}\right)$ and choose a sequence of positive numbers $\left\{s_{n}\right\}$, strictly increasing to $\infty$, such that

$$
f\left(A_{0} s_{n}\right) \geq B f\left(s_{n}\right), \quad n \geq 1 .
$$

Upon defining $\left\{u_{n}\right\}$ and $\left\{v_{n}\right\}$ as in the proof of Lemma 5.1, we have

$$
f\left(A_{0} f^{\sim}\left(v_{n}\right)\right) \geq f\left(A_{0} s_{n}\right) \geq B f\left(s_{n}\right) \geq B f\left(f^{\sim}\left(u_{n}\right)\right),
$$

and, in view of (22) and (25),

$$
c_{0} \geq \limsup _{n \rightarrow \infty} \frac{f\left(A_{0} f^{\sim}\left(v_{n}\right)\right)}{v_{n}} \geq B \limsup _{n \rightarrow \infty} \frac{f\left(f^{\sim}\left(v_{n}\right)\right)}{v_{n}}=B .
$$

This contradiction proves that $f^{\sim} \in \mathcal{Q} \mathcal{V}_{1} \Longrightarrow f \in \mathcal{O S} \mathcal{S}$.

To prove the converse, let $f \in \mathcal{O S} \mathcal{V}$ and $f^{\sim} \notin \mathcal{Q} \mathcal{V}_{1}$. Then there exists a number $c_{0} \geq 1$ such that $f^{*}(A) \leq c_{0}$ for every $A>0$. Now, $f^{\sim} \notin \mathcal{Q} \mathcal{V}_{1}$ implies that, for any given $c>1$,

$$
\liminf _{t \rightarrow \infty} \frac{f^{\sim}(c t)}{f^{\sim}(t)}<\infty
$$

Then we find $B>0$ such that

$$
f^{\sim}(c t) \leq B f^{\sim}(t)
$$

for infinitely many large $t$. On choosing $c>c_{0}$, we get

$$
c=\lim _{t \rightarrow \infty} \frac{f\left(f^{\sim}(c t)\right)}{t} \leq \liminf _{t \rightarrow \infty} \frac{f\left(B f^{\sim}(t)\right)}{f\left(f^{\sim}(t)\right)} \leq \limsup _{s \rightarrow \infty} \frac{f(B s)}{f(s)} \leq c_{0} .
$$

This contradiction proves that $f \in \mathcal{O} S \mathcal{V} \Longrightarrow f^{\sim} \in \mathcal{Q} \mathcal{V}_{1}$.

Thus, statement (vi) is proved.

\section{ASYMPtotic RIGHT AND LEFT QUASI-INVERSE FUNCTIONS}

Let $f \in \mathbb{F}^{(\infty)}$. As discussed above, an asymptotic quasi-inverse function $f^{\sim}$ for a given function $f$ is characterized by the property

$$
\left(f \circ f^{\sim}\right)(t) \sim t \quad \text { as } t \rightarrow \infty,
$$

where the symbol $\circ$ stands for the superposition of functions. For obvious reasons, any function $f_{r}^{\sim}$ satisfying (26), with $f_{r}^{\sim}$ instead of $f^{\sim}$ and such that $f_{r}^{\sim}(t) \rightarrow \infty$ as $t \rightarrow \infty$, can be called an asymptotic right quasi-inverse function (of $f$ ). 
Another asymptotic property, inherited from the definition of an inverse function, is that

$$
(f \tilde{\ell} \circ f)(t) \sim t \quad \text { as } t \rightarrow \infty
$$

Any $f_{\ell} \tilde{\ell}$ satisfying (27) and such that

$$
f_{\ell}(t) \rightarrow \infty \quad \text { as } t \rightarrow \infty
$$

may be called an asymptotic left quasi-inverse function (of $f$ ). The generalized inverse function $f^{\leftarrow}$, for example, is one version of both, an asymptotic right quasi-inverse function of $f$ if $f$ is continuous, and an asymptotic left quasi-inverse function of $f$ if $f$ is strictly increasing.

Generally, if $g$ is an asymptotic right quasi-inverse function of $f$, then $f$ is an asymptotic left quasi-inverse function of $g$, and vice versa. In view of this symmetry, Theorems 4.2 4.5 can be reformulated as follows.

Theorem 6.1. Let both functions $f$ and $f_{\ell}$ be nondecreasing. Then

(a) $f \in \mathcal{P J} \Longleftrightarrow f_{\ell} \in \mathcal{O} \mathcal{N} \mathcal{O}$;

(b) $f \in \mathcal{O R V} \Longleftrightarrow f_{\ell} \in \mathcal{P J}$;

(c) $f \in \mathcal{O} \mathcal{R} \mathcal{P} \cap \mathcal{P J} \Longleftrightarrow f_{\ell} \in \mathcal{O} \mathcal{R V} \cap \mathcal{P J}$.

Theorem 6.2. Let both functions $f$ and $f_{\ell}$ be nondecreasing. Then

(a) $f \in \mathcal{R} \mathcal{V}_{\infty} \Longleftrightarrow f_{\ell} \in \mathcal{R} \mathcal{V}_{0}$;

(b) $f \in \mathcal{R} \mathcal{V}_{0} \Longleftrightarrow f_{\ell} \in \mathcal{R} \mathcal{V}_{\infty}$.

Theorem 6.3. Let both functions $f$ and $f_{\ell}$ be nondecreasing. Then

(a) $f \in \mathcal{O S V} \Longleftrightarrow f_{\ell} \in \mathcal{Q} \mathcal{V}_{1}$;

(b) $f \in \mathcal{Q V} \mathcal{V}_{1} \Longleftrightarrow f_{\ell}^{\sim} \in \mathcal{O S V}$.

\section{BIBLIOGRAPHY}

1. S. Aljančić and D. Arandelović, O-regularly varying functions, Publ. Inst. Math. (Beograd) (N.S.) 22 (36) (1977), 5-22. MR0466438 (57:6317)

2. V. G. Avakumović, Über einen O-Inversionssatz, Bull. Int. Acad. Youg. Sci. 29-30 (1936), $107-117$.

3. N. K. Bari and S. B. Stechkin, Best approximation and differential properties of two conjugate functions, Trudy Mosk. Mat. Obsch. 5 (1956), 483-522. (Russian) MR0080797 (18:303e)

4. S. M. Berman, Sojourns and extremes of a diffusion process on a fixed interval, Adv. Appl. Prob. 14 (1982), 811-832. MR677558 (84d:60113)

5. S. M. Berman, The tail of the convolution of densities and its application to a model of HIVlatency time, Ann. Appl. Prob. 2 (1992), 481-502. MR1161063 (94b:60020)

6. N. H. Bingham and C. M. Goldie, Extensions of regular variation, I, II, Proc. London. Math. Soc. 44 (1982), 473-534. MR656246 (83m:26004a)

7. N. H. Bingham, C. M. Goldie, and J. L. Teugels, Regular Variation, Cambridge University Press, Cambridge, 1987. MR898871 (88i:26004)

8. V. V. Buldygin, O. I. Klesov, and J. G. Steinebach, Properties of a subclass of Avakumović functions and their generalized inverses, Ukrain. Math. J. 54 (2002), 179-206. MR1952816 (2003i:60044) 
9. V. V. Buldygin, O. I. Klesov, and J. G. Steinebach, On factorization representations for Avakumović-Karamata functions with nondegenerate groups of regular points, Anal. Math. 30 (2004), 161-192. MR2093756 (2005f:26029)

10. V. V. Buldygin, O. I. Klesov, and J. G. Steinebach, On some properties of asymptotically quasiinverse functions and their applications I, Teor. Imov. Mat. Stat. 70 (2004), 9-25; English transl. in Theory Probab. Math. Statist. 70 (2005), 11-28. MR2109819 (2005i:26005)

11. V. V. Buldygin, O. I. Klesov, and J. G. Steinebach, On some properties of asymptotically quasiinverse functions and their applications II, Teor. Imov. Mat. Stat. 71 (2004), 34-48; English transl. in Theory Probab. Math. Statist. 71 (2005), 37-52. MR2144319 (2006d:26002)

12. V. V. Buldygin, O. I. Klesov, and J. G. Steinebach, The PRV property of functions and the asymptotic behavior of solutions of stochastic differential equations, Teor. Imov. Mat. Stat. $\mathbf{7 2}$ (2005), 10-23; English transl. in Theory Probab. Math. Statist. 72 (2006), 11-25. MR2168132 (2006e:60079)

13. V. V. Buldygin, O. I. Klesov, and J. G. Steinebach, On some extensions of Karamata's theory and their applications, Publ. Inst. Math. (Beograd) (N.S.) 80(94) (2006), 59-96. MR.2281907 $(2007 \mathrm{j}: 26001)$

14. D. B. H. Cline, Intermediate regular and П-variation, Proc. London Math. Soc. 68 (1994), 594-616. MR 1262310 (95c:26001)

15. D. Djurčić, O-regularly varying functions and strong asymptotic equivalence, J. Math. Anal. Appl. 220 (1998), 451-461. MR 1614959 (99c:26002)

16. D. Djurčić and A. Torgašev, Strong asymptotic equivalence and inversion of functions in the class $K_{c}$, J. Math. Anal. Appl. 255 (2001), 383-390. MR.1815787 (2001m:26005)

17. D. Drasin and E. Seneta, A generalization of slowly varying functions, Proc. Amer. Math. Soc. 96 (1986), no. 3, 470-472. MR822442 (87d:26002)

18. W. Feller, One-sided analogues of Karamata's regular variation, L'Enseignement Math. 15 (1969), 107-121. MR0254905(40:8112)

19. I. V. Grinevich and Yu. S. Khokhlov, The domains of attraction of semistable laws, Teor. Veroyatnost. Primenen. 40 (1995), no. 2, 417-422; English transl. in Theory Probab. Appl. 40 (1995), no. 2, 361-366. MR1346477 (96k:60035)

20. A. Gut, O. Klesov, and J. Steinebach, Equivalences in strong limit theorems for renewal counting processes, Statist. Probab. Lett. 35 (1997), 381-394. MR.1483025 (98m:60043)

21. L. de Haan and U. Stadtmüller, Dominated variation and related concepts and Tauberian theorems for Laplace transformations, J. Math. Anal. Appl. 108 (1985), 344-365. MR793651 (86k:44002)

22. J. Karamata, Sur un mode de croissance régulière des fonctions, Mathematica (Cluj) 4 (1930), 38-53.

23. J. Karamata, Sur un mode de croissance régulière. Théorèmes fondamenteaux, Bull. Soc. Math. France 61 (1933), 55-62. MR1504998

24. J. Karamata, Bemerkung über die vorstehende Arbeit des Herrn Avakumović, mit näherer Betrachtung einer Klasse von Funktionen, welche bei den Inversionssätzen vorkommen, Bull. Int. Acad. Youg. Sci. 29-30 (1936), 117-123.

25. O. Klesov, Z. Rychlik, and J. Steinebach, Strong limit theorems for general renewal processes, Theory Probab. Math. Statist. 21 (2001), 329-349. MR1911442 (2003j:60120)

26. B. H. Korenblyum, On the asymptotic behaviour of Laplace integrals near the boundary of a region of convergence, Dokl. Akad. Nauk. USSR (N.S.) 104 (1956), 173-176. MR0074550 $(17: 605 \mathrm{a})$

27. W. Matuszewska, On a generalization of regularly increasing functions, Studia Math. 24 (1964), 271-279. MR0167574 (29:4846)

28. W. Matuszewska and W. Orlicz, On some classes of functions with regard to their orders of growth, Studia Math. 26 (1965), 11-24. MR0190273 (32:7686)

29. S. Resnick, Extreme Values, Regular Variation, and Point Processes, Springer-Verlag, New York, 1987. MR 900810 (89b:60241)

30. B. A. Rogozin, A Tauberian theorem for increasing functions of dominated variation, Sibirsk. Matem. Zh. 43 (2002), 442-445; English transl. in Siberian Math. J. 43 (2002), 353-356. MR.1902831 (2003f:40010)

31. E. Seneta, Regularly Varying Functions, Springer-Verlag, Berlin, 1976. MR0453936 (56:12189)

32. U. Stadtmüller and R. Trautner, Tauberian theorems for Laplace transforms, J. Reine Angew. Math. 311/312 (1979), 283-290. MR549970 (81f:44006)

33. U. Stadtmüller and R. Trautner, Tauberian theorems for Laplace transforms in dimension $D>1$, J. Reine Angew. Math. 323 (1981), 127-138. MR611447(82i:44001) 
34. A. L. Yakymiv, Asymptotics properties of the state change points in a random record process, Teor. Veroyatnost. Primenen. 31 (1986), 577-581; English transl. in Theory Probab. Appl. 31 (1987), 508-512. MR866880 (88b:60093)

35. A. L. Yakymiv, Asymptotics of the probability of nonextinction of critical Bellman-Harris branching processes, Proc. Steklov Inst. Math. 4 (1988), 189-217. MR0840684|(88d:60221)

Department of Mathematical Analysis and Probability Theory, National Technical University of Ukraine "KPI", Peremogy Avenue 37, Kyiv 03056, Ukraine

E-mail address: valbuld@comsys.ntu-kpi.kiev.ua

Department of Mathematical Analysis and Probability Theory, National Technical University of Ukraine "KPI", Peremogy Avenue 37, Kyiv 03056, Ukraine

E-mail address: klesov@math.uni-paderborn.de

Universität zu Köln, Mathematisches Institut, Weyertal 86-90, D-50931 Köln, Germany

E-mail address: jost@math.uni-koeln.de

Received 25/DEC/2006

Translated by THE AUTHORS 